\title{
Editorial
}

\section{Recent Trends in Dynamical Systems on Time Scales}

\author{
George Eid, ${ }^{1}$ Jeffry Neugebauer $\mathbb{D}^{1},{ }^{2}$ Youssef Naim Raffoul $\mathbb{D}^{\circ},{ }^{3}$ and Cemil Tunç $\mathbb{C}^{4}$ \\ ${ }^{1}$ Faculty of Natural \& Applied Sciences, Notre Dame University-Louaize, Lebanon \\ ${ }^{2}$ Eastern Kentucky University, Department of Mathematics, USA \\ ${ }^{3}$ University of Dayton, Department of Mathematics, Dayton Ohio 45469-2316, USA \\ ${ }^{4}$ Department of Mathematics, Faculty of Sciences, Yuzuncu Yil University, Turkey \\ Correspondence should be addressed to Youssef Naim Raffoul; yraffoull@udayton.edu
}

Received 20 June 2018; Accepted 20 June 2018; Published 19 July 2018

Copyright (C) 2018 George Eid et al. This is an open access article distributed under the Creative Commons Attribution License, which permits unrestricted use, distribution, and reproduction in any medium, provided the original work is properly cited.

The theory of time scales was created to unify continuous and discrete analysis. Difference and differential equations can be studied simultaneously by studying dynamic equations on time scales. Recently, there has been much interest in the study of dynamical systems on time scales due to their applications to real world problems, such as electric circuits and insect populations. Many application problems can be studied more precisely using dynamical systems on time scales. Subjects such as existence and uniqueness of solutions, stability, Floquet theory, periodicity, stability, and boundedness of solutions can be studied more precisely and generally by utilizing dynamical systems on time scales. Recently, many researchers have been looking at the applications of time scales in various economics models utilizing optimal control theory and developing more realistic models in economics and population dynamics using time scales.

This special issue places its emphasis on the study of the applications of dynamical system on time scales; such applications include economics models utilizing optimal control theory, fractional calculus, and the development of new population models.

All manuscripts submitted to this special issue went through a thorough peer-refereeing process. Based on the reviewers' reports, we collected 5 original research articles by more than 17 active international researchers on dynamical systems.

To be more comprehensive, we give a description of each article in this special issue by providing a short editorial note summarizing each paper.

W. Kumam et al. established sufficient conditions for existence of solutions to the coupled systems of higher-order hybrid fractional differential equations with three-point boundary conditions. They apply the coupled fixed point theorem of Krasnoselskii type to form adequate conditions for existence of solutions to the proposed system. They provided a nice example as an application.

Z. Wang et al. proposed a new docking system that is more efficient and requires less makespan. A cross-docking system is proposed with multiple receiving and shipping dock doors. Their objective is to find the best door assignments and the sequences of trucks in the principle of products distribution to minimize the total makespan of cross-docking. To solve the problem that is regarded as a mixed integer linear programming (MILP) model, three metaheuristics, namely, harmony search (HS), improved harmony search (IHS), and genetic algorithm (GA), are proposed. Furthermore, the fixed parameters are optimized by Taguchi experiments to improve the accuracy of solutions further. Finally, they ended the paper with several numerical examples to evaluate the performances of proposed algorithms.

Z. Liu et al. proposed a new fractional two-dimensional triangle function combination discrete chaotic map (2DTFCDM) with the discrete fractional difference. Bifurcation behaviors, drawing the bifurcation diagrams, the largest Lyapunov exponent plot, and the phase portraits of the proposed map, were studied. On the application side, they apply the proposed discrete fractional map into image encryption with the secret keys ciphered by Menezes-Vanstone Elliptic Curve Cryptosystem (MVECC). Finally, the image encryption algorithm is analyzed in four main aspects that indicate that the proposed algorithm is better than others.

$Z$. Wei et al. studied the dynamics of a stochastic modified Bazykin predator-prey system with Lévy jumps. First, they show that the system has a unique global positive solution and 
discovered new properties of solutions. Then, some sufficient conditions for persistence and extinction are derived by Itô formula and some inequalities on stochastic analysis. Numerical simulations are provided to check the main results.

$\mathrm{Z}$. Li and D. Tan established an analytical framework studying the optimal dynamic pricing and advertising strategies for online providers; it shows how the strategies are influenced by the videos available time and the viewers' emotional factor. They create the two-stage strategy of revenue models involving a single fee mode and a mixed fee-free mode and find out the optimal fee charge and advertising level of online video services. According to the results, the optimal video price and ads volume dynamically vary over time. The viewer's aversion level to advertising has direct effects on both the volume of ads and the number of viewers who have selected low-quality content. The optimal volume of ads decreases with the increase of ads-aversion coefficient, while it increases as the quality of videos increases. The results also indicate that, in the long run, a pure fee mode or free mode is the optimal strategy for online providers.

\section{Acknowledgments}

We would like to thank the editorial board members of this journal, for their support in making this special issue a reality.

George Eid

Jeffrey Neugebauer

Youssef Naim Raffoul

Cemil Tunç 


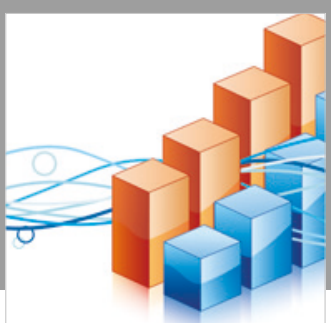

Advances in

Operations Research

\section{-n-m}
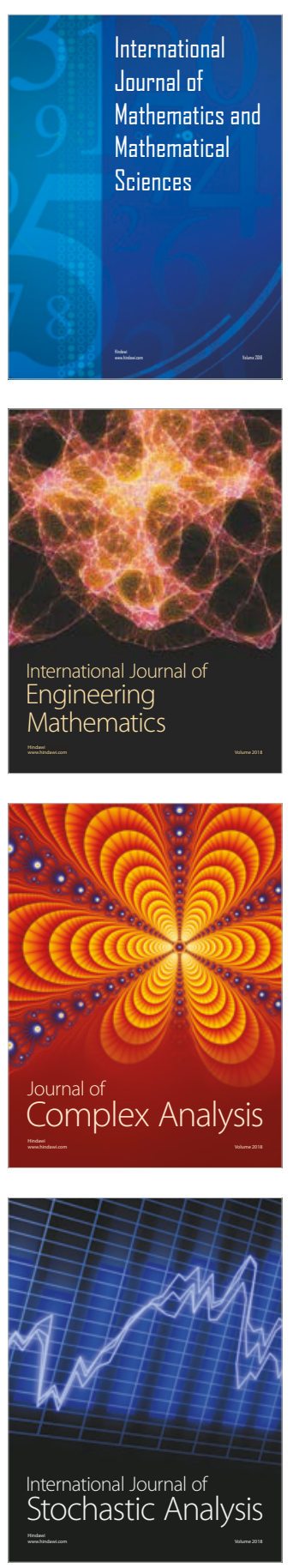
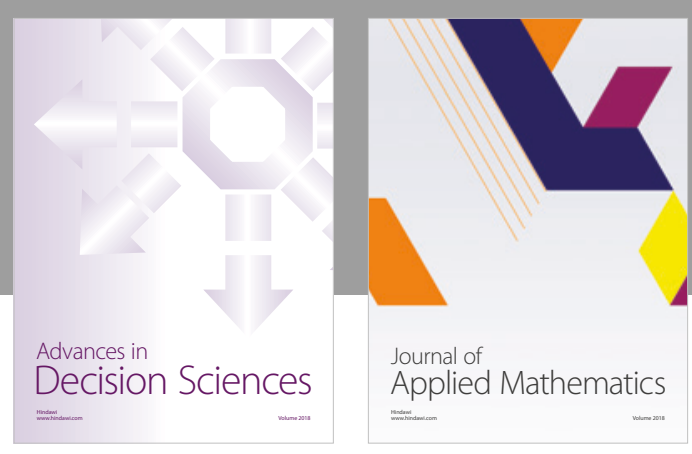

Journal of

Applied Mathematics
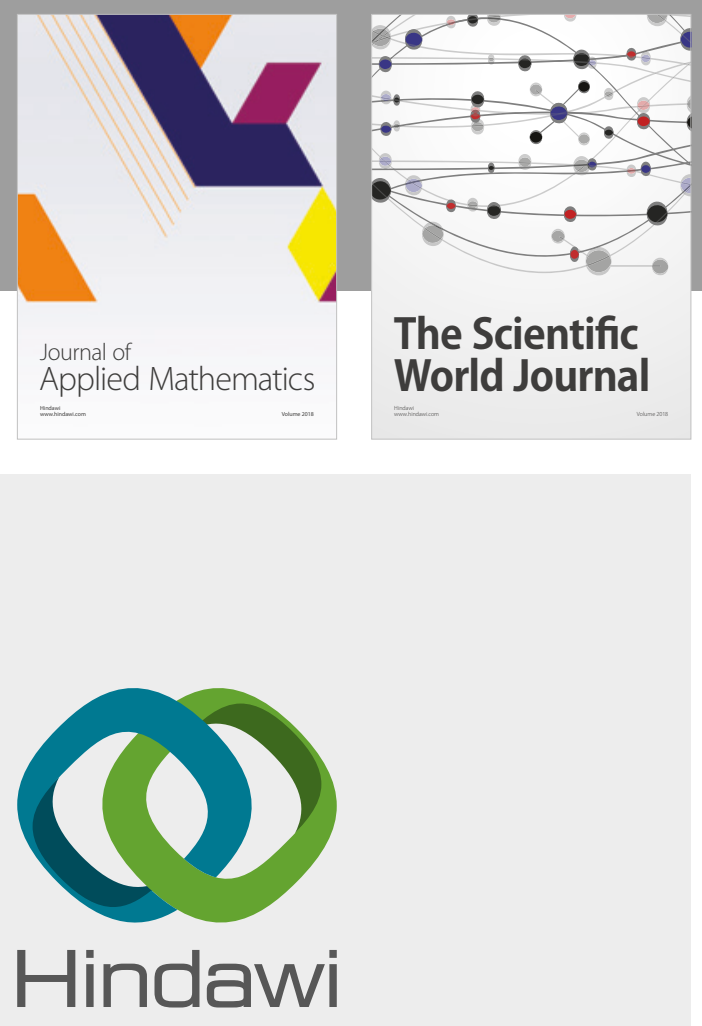

Submit your manuscripts at

www.hindawi.com

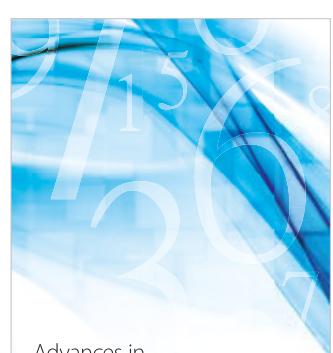

Advances in
Numerical Analysis
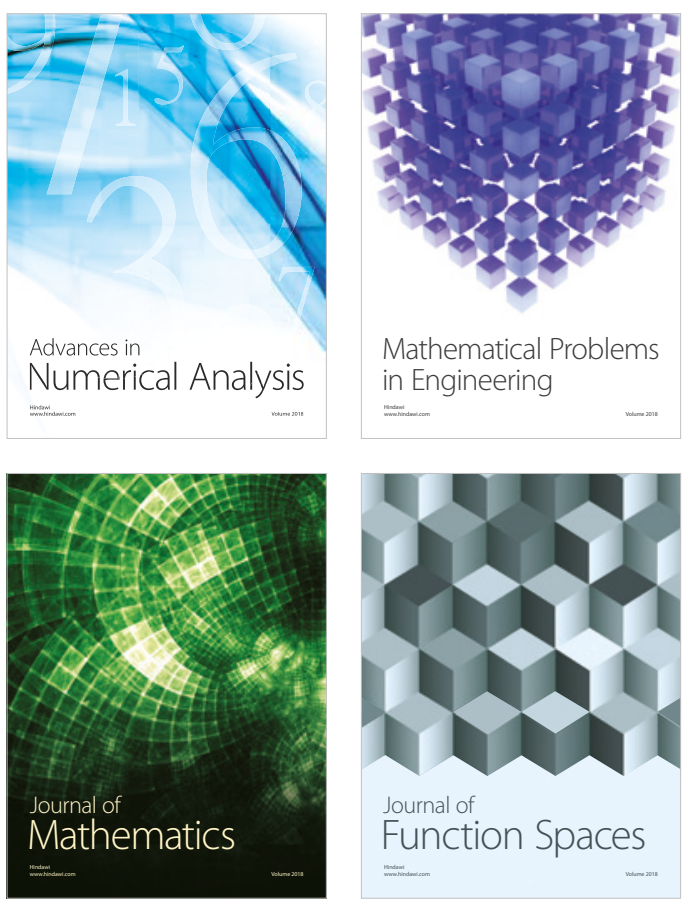

Mathematical Problems in Engineering

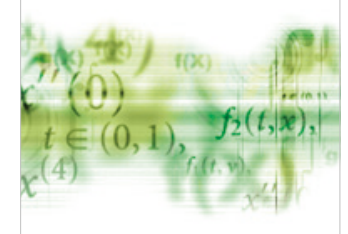

International Journal of

Differential Equations

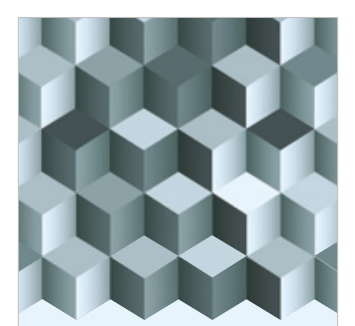

Journal of

Function Spaces

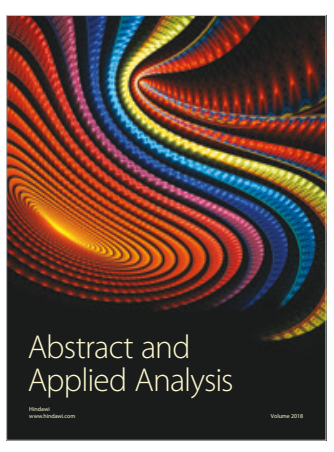

The Scientific

World Journal

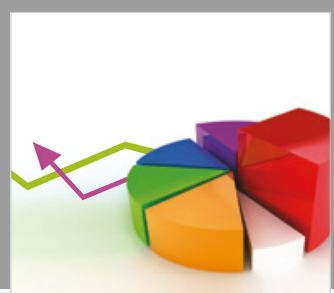

Journal of

Probability and Statistics
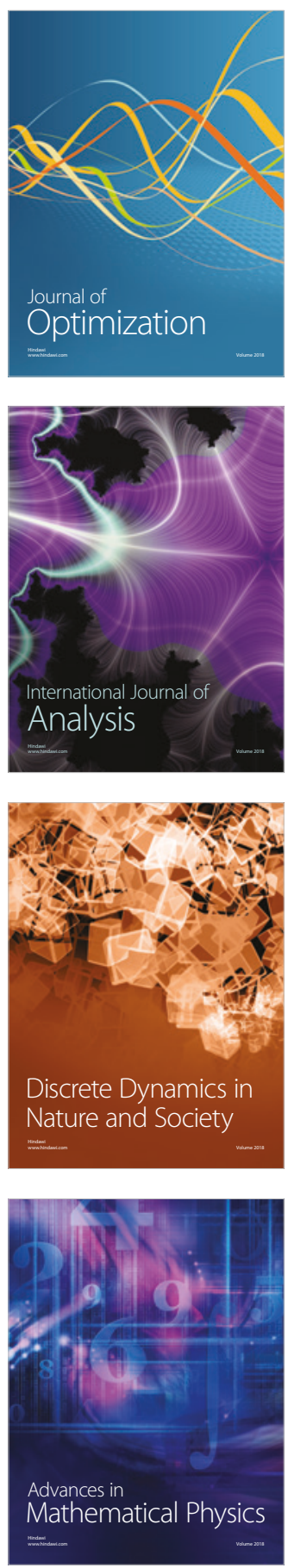